\title{
Consequências das Quedas em Idosos Vivendo na Comunidade
}

\author{
Consequences of falls in older people living in the community
}

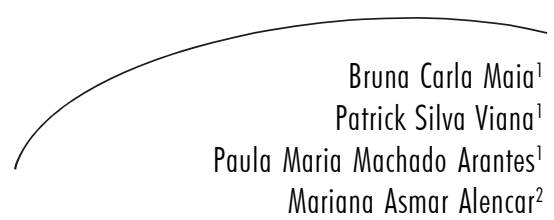

Resumo

As quedas em idosos são um problema frequente, com importantes consequências físicas, psicológicas e sociais. O objetivo deste estudo foi realizar uma revisão sistematizada da literatura sobre as consequências das quedas em idosos vivendo na comunidade. A busca foi realizada entre junho a outubro de 2008 nas bases de dados eletrônicas Medline, Lilacs, PEDro e SciELO. Apenas artigos em inglês, português e espanhol foram considerados para esta revisão. Foram identificados inicialmente 114 artigos potencialmente relevantes. Após análise do título e dos resumos, considerando-se critérios de inclusão e exclusão predeterminados, foram selecionados para esta revisão dez artigos. Foram identificadas as seguintes consequências: fraturas, imobilização, lesões de tecidos moles, contusões, entorses, feridas e abrasões, lesões musculares e neurológicas, surgimento de outras doenças, dor, declínio funcional e da atividade física, atendimento médico, hospitalização, reabilitação, medo de cair, abandono de atividades, tristeza, mudança na vida/comportamentos, sentimento de impotência, declínio em atividade social, perda de autonomia e da independência, mudança de domić́lio/ ambiente, rearranjo familiar e morte. As fraturas e o medo de uma nova queda ficaram dentre as consequências mais citadas. O conhecimento das consequências físicas, psicológicas e sociais das quedas em idosos é de extrema importância, pois ele auxiliará no delineamento das estratégias preventivas e de reabilitação de tais repercussões.

\section{Abstract}

The falls in the elderly are a frequent problem with significant physical, psychological and social consequences. The aim of this study was to make a systematic review about the consequences of falls in older people living in the community. The search was conducted between June and October 2008 in electronic databases Medline, Lilacs, PEDro and SciELO. Only articles in English, Portuguese and Spanish were considered for this review. It were found the following

Palavras-chave: Idosos. Acidentes por Quedas. Saúde do idoso. Prevenção de acidentes.

Key words: Elderly. Accidental Falls. Health of the Elderly. Accident Prevention.

\footnotetext{
Fundação Comunitária de Ensino Superior de Itabira. Itabira, MG, Brasil.
}

2 Universidade FUMEC. Belo Horizonte, MG, Brasil. 
consequences: fractures, immobilization, soft-tissue injuries, contusions, sprains, wounds and abrasions, muscle and neurological injuries, occurrence of other diseases, pain, functional and physical activity decline, medical care, hospitalization, rehabilitation, fear of falling, restriction of activities, sadness, behavior change, feelings of powerlessness, decline in social activity, loss of autonomy and independence, change of home/environment, family rearrangement and death. Fractures and fear of falling were among the most cited consequences. The knowledge about physical, psychological and social consequences of falls in elderly people is important, because it will help to delineate preventive and rehabilitation strategies of these repercussions.

\section{INTRODUÇÃO}

O envelhecimento é um processo dinâmico e progressivo, no qual há alterações morfológicas, funcionais e bioquímicas, com redução na capacidade de adaptação homeostática às situações de sobrecarga funcional, alterando progressivamente o organismo e tornando-o mais susceptível às agressões intrínsecas $\mathrm{e}$ extrínsecas. ${ }^{1}$ Representa a passagem do tempo, não a patologia, ${ }^{2}$ sendo um processo natural e fisiológico, no qual as experiências emocionais, psicológicas e ambientais o tornam singular e individual. ${ }^{3}$ Desta forma, duas pessoas não envelhecem de maneira idêntica. ${ }^{4}$

Nas últimas décadas, observou-se um nítido processo de envelhecimento demográfico. A Organização das Nações Unidas (ONU) considera o período de 1975 a 2025 a Era do Envelhecimento. ${ }^{5}$ No Brasil, a expectativa do Instituto Brasileiro de Geografia e Estatística (IBGE) é de que a população com mais de 60 anos de idade seja aproximadamente $11 \%$ da população geral até o ano de 2020.6 O índice de envelhecimento mostra que para cada 100 indivíduos jovens, existem 35,4 acima de 60 anos. A esperança de vida, que era em torno de 33,7 anos em 1950/1955, passou para 50,99 em 1990, chegou até 66,25 em 1995 e deverá alcançar 77,08 em 2020/2025. ${ }^{5}$

Esse processo de envelhecimento demográfico repercutiu e continua repercutindo nas diferentes esferas da estrutura social, econômica, política e cultural da sociedade, uma vez que os idosos, da mesma forma que os demais segmentos etários (crianças, jovens e adultos), possuem demandas específicas para obtenção de adequadas condições de vida. ${ }^{5}$ Tais demandas têm despertado grande interesse na área da saúde pública. ${ }^{6}$

Uma das grandes preocupações relacionadas ao envelhecimento é que o aumento da expectativa de vida está associado a uma alta taxa de comorbidades. ${ }^{8}$ A instabilidade postural e as quedas fazem parte das síndromes geriátricas que englobam as alterações de saúde mais comuns nos idosos, ${ }^{9}$ constituindo um dos principais problemas clínicos e de saúde pública devido à sua alta incidência, às consequentes complicações para a saúde e aos altos custos assistenciais. ${ }^{10}$

Queda pode ser definida como "um evento não intencional que tem como resultado a mudança de posição do indivíduo para um nível mais baixo, em relação a sua posição inicial". ${ }^{11}$ Alguns autores referem-se à queda como uma síndrome geriátrica por ser considerado um evento multifatorial e heterogêneo. ${ }^{12}$

Aproximadamente $30 \%$ dos indivíduos com mais de 65 anos de idade caem ao menos uma vez por ano, dos quais a metade de forma recorrente. ${ }^{13,14}$ Pessoas de todas as idades apresentam risco de sofrer queda. Porém, para os idosos, elas possuem um significado muito relevante, pois podem levá-lo à incapacidade, injúria e morte. Seu custo social é imenso e se torna maior quando o idoso tem diminuição da autonomia e da independência ou passa a necessitar de institucionalização. ${ }^{15}$ 
As quedas geralmente têm etiologia multifatorial e seus fatores causadores são classificados como: intrínsecos, ou seja, os decorrentes de alterações fisiológicas relacionadas ao envelhecimento, a doenças e efeitos causados pelo uso de fármacos; e extrínsecos, que são fatores que dependem de circunstâncias sociais e ambientais que criam desafios ao idoso. ${ }^{15}$ Estes fatores interagem como agentes determinantes e predisponentes, tanto para quedas acidentais quanto para quedas recorrentes. Esta complexidade da etiologia das quedas, associada às graves consequências geradas por estas, impõem aos profissionais de saúde o grande desafio de identificar os possíveis fatores de risco e tratar os fatores etiológicos e comorbidades presentes. ${ }^{16}$ A importância da identificação de tais fatores de risco é reforçada pelo maior sucesso das intervenções que se baseiam na identificação precoce dos idosos com maior chance de sofrerem quedas e particularmente aqueles que, além do risco de queda, apresentem também risco aumentado de sofrerem lesões graves decorrentes da mesma. ${ }^{17-19}$

As quedas em idosos são um problema frequente com importantes consequências físicas, psicológicas e sociais. ${ }^{20}$ Dentre as principais consequências decorrentes das quedas, encontram-se as fraturas, que parecem imputar ao idoso maior vulnerabilidade a novos episódios, independentemente de sua frequência. ${ }^{21}$ Entre outras consequências das quedas, encontram-se lesões na cabeça, ferimentos graves, ansiedade, depressão e o chamado "medo de cair" (medo de subsequentes quedas), que também pode acometer idosos que nunca caíram. ${ }^{10}$

Do ponto de vista econômico, o custo por qualquer problema de saúde pode ser classificado em duas grandes categorias: custos diretos e indiretos. Os custos diretos dizem respeito aos custos médicos e não-médicos "relacionados ao diagnóstico, tratamento, recuperação e reabilitação da doença”. Os custos indiretos referem-se à perda de produção e produtividade trazida pelo problema de saúde. ${ }^{22}$ De acordo com Jorge e Koizumi, as quedas geram um custo hospitalar no estado de São Paulo de aproximadamente $\mathrm{R} \$ 122,55$ por dia.
A cada ano, o Sistema Único de Saúde (SUS) tem gastos crescentes com tratamentos de fraturas decorrentes das quedas. Em 2006 foram gastos R\$ 49.884.326 com internações de idosos por fratura de fêmur e R $\$ 20$ milhões com medicamentos. ${ }^{23}$

Além das consequênciasfísicas, atualmentetem sido dado enfoque às repercussões psicológicas e sociais que as quedas trazem. As quedas, além de produzirem importante perda de autonomia e qualidade de vida entre idosos, podem também repercutir entre seus cuidadores, principalmente familiares, que devem se mobilizar em torno de cuidados especiais, adaptando toda sua rotina em função da recuperação ou adaptação do idoso após a queda. ${ }^{24}$

O conhecimento das consequências físicas, psicológicas e sociais das quedas em idosos é de extrema importância, pois ele auxiliará no delineamento das estratégias preventivas e de reabilitação de tais repercussões. Assim, o objetivo deste estudo foi realizar uma revisão bibliográfica sistemática da literatura através da seleção e análise criteriosa de artigos científicos que investigaram as consequências das quedas em idosos vivendo na comunidade.

\section{MATERIAIS E MÉTODOS}

\section{Estratégias de busca}

A busca foi realizada nas bases de dados eletrônicas Medline (National Library of Medicine), Lilacs (Literatura Latino-americana e do Caribe em Ciências da Saúde), PEDro (Physiotherapy Evidence Database) e SciELO (Scientific Electronic Library Online). Foram utilizados os descritores: idosos, quedas e consequências, seus equivalentes em inglês (elderly, older, aged, falls e consequences) e seus equivalentes em espanhol (anciano, caídas e consecuencias). Durante a busca, houve o cruzamento dessas palavras.

Apenas artigos em inglês, português e espanhol foram considerados para esta revisão. Além do idioma de publicação, foram utilizadas como limite na estratégia de busca, as palavraschave pesquisadas constarem no título ou resumo. 
Seleção dos estudos

Foram selecionados para esta revisão estudos publicados no período de 1998 a 2008. Em relação aos participantes, foram selecionados apenas estudos com idosos (maiores que 60 anos), vivendo na comunidade. $\mathrm{O}$ desfecho considerado foi consequências das quedas. Os artigos que obedeceram aos critérios de inclusão foram analisados independentemente por dois revisores.

\section{Análise dos artigos}

A busca foi realizada por dois revisores, que avaliaram os títulos e resumos independentemente; se existisse alguma discordância entre estes quanto à seleção dos artigos, esta seria resolvida por um terceiro revisor. Após esta primeira seleção, os artigos foram lidos na íntegra e aqueles que não estivessem dentro dos critérios estabelecidos foram excluídos do estudo.

\section{Descrição dos artigos}

Para organizar as informações dos artigos, foi realizada uma síntese das principais informações de cada estudo em uma tabela que continha os seguintes tópicos: autor(es) e ano, participantes, Instrumentos de avaliação e resultados consequências.

\section{RESULTADOS}

\section{Estudos Identificados}

$\mathrm{Na}$ pesquisa inicial, realizada nas bases de dados eletrônicas MEDLINE, LILACS, PEDro e SciELO, foram identificados, respectivamente, em cada uma delas, 102, sete, dois, três artigos potencialmente relevantes. Após análise do título, foram excluídos 72 artigos do MEDLINE, seis do LILACS, dois do PEDro e um no SciELO. Após a leitura dos resumos, foram excluídos 23 artigos do Medline, por não abordarem especificamente o desfecho consequências das quedas, por estudarem apenas idosos institucionalizados ou por serem artigos de revisão. Assim, conforme os critérios de inclusão e exclusão predeterminados, foram selecionados para o estudo dez artigos, sendo sete artigos do MEDLINE, um do LILACS e dois do SciELO.

\section{Características gerais dos artigos encontrados}

De acordo com os artigos selecionados para esta revisão sistemática, foram identificadas as seguintes consequências: fraturas, ${ }^{15,20,25-31}$ imobilização, ${ }^{15,20,25,32}$ lesões de tecidos moles, ${ }^{27,28}$ contusões, ${ }^{15,29}$ entorses, ${ }^{26,30}$ feridas, ${ }^{20,26,29,30 \text {, }}$ abrasões, ${ }^{27}$ extravasamento de líquido, ${ }^{30}$ lesões musculares, ${ }^{30}$ lesões neurológicas, ${ }^{15,25,30,31}$ enfermidade nos pés, ${ }^{20}$ surgimento de outras doenças, ${ }^{15}$ doenças sensoriais, ${ }^{15}$ danos físicos, ${ }^{30}$ dor, ${ }^{28,30}$ dificuldade para utilização dos membros superiores (MMSS), ${ }^{20}$ dificuldade para levantar da cadeira e realizar exercícios, ${ }^{20}$ dificuldade para andar, ${ }^{20,30}$ declínio da atividade funcional e atividade física, ${ }^{30}$ dificuldade nas atividades de vida diária (AVDs), ${ }^{15,20,27,30}$ atendimento médico e de urgência, ${ }^{25-27,30,32}$ hospitalização, ${ }^{15,31}$ medicação, ${ }^{30}$ reabilitação, ${ }^{30}$ fisioterapia, ${ }^{30}$ cuidados $\mathrm{da}$ enfermagem ${ }^{30}$ e cirurgias. $^{30}$ Foram encontradas nos estudos também consequências psicológicas e sociais, como medo de cair, ${ }^{15,20,25,26,32}$ abandono de atividades, ${ }^{15,25}$ tristeza, ${ }^{32}$ mudança na vida/ comportamentos, ${ }^{20,28}$ problemas de memória, ${ }^{20}$ problemas para se orientar no espaço e tempo, ${ }^{20}$ sentimento de impotência, ${ }^{32}$ declínio em atividade social, ${ }^{30}$ isolamento, ${ }^{15}$ perda de autonomia,,${ }^{15}$ liberdade pessoal e independência, ${ }^{32}$ mudança de domicilio/ambiente, ${ }^{15,25,28,30}$ modificações de hábitos, ${ }^{15,25}$ volta da independência, ${ }^{27}$ atitude protetora, ${ }^{15}$ rearranjo familiar e morte. ${ }^{15}$

Dentre as consequências encontradas neste estudo, as mais citadas foram as fraturas, que variaram de $2,56^{26}$ a $64 \%,{ }^{15}$ sendo que as mais comuns ocorreram no fêmur, variando entre $33,3^{25}$ a $62 \%,{ }^{15}$ quadril $2,6^{26}$ a $12 \%,{ }^{31}$ braços $7^{29}$ a $49 \%{ }^{26}$ e antebraço $9,9^{29}$ a $12,5 \%,{ }^{15}$ considerandose as fraturas de rádio. A ocorrência foi maior em mulheres do que em homens. ${ }^{15,26-29}$

O medo de uma nova queda também teve alta frequência entre os estudos, sendo identificado com uma taxa de variação entre $44^{15}$ a $88,5 \% .{ }^{25} \mathrm{O}$ 
abandono de atividades teve uma variação de $12^{15}$ a $25,9 \% .{ }^{25}$ A perda de autonomia foi encontrada em um estudo (14\%). ${ }^{15}$

Os números de lesões, torções, contusões, ferimentos (superficiais) e surgimento de outras doenças foram citados na maioria dos artigos presentes nesta revisão, com uma variação de $1^{30}$ a $56 \%{ }^{20}$

A procura de atendimento de emergência / médico foi frequentemente citada, com uma variação de $7^{27}$ a $48,6 \% .{ }^{25}$

Entre as limitações funcionais encontradas, destacam-se: dificuldade para utilizar os membros superiores (MMSS), dificuldade de levantar da cadeira, de realizar exercícios, de caminhar em superfície plana, de deitar e levantar da cama, vestir-se, caminhar fora de casa, com uma variação $6,9^{30}$ a $40,9 \% .{ }^{20}$

A mudança de domicílio/ambiente foi identificada nos estudos com uma variação de $0,5^{30}$ a $64 \% .^{28}$

A dor pós-queda variou entre $4,9^{30}$ a $31 \%^{28}$.

A morte foi encontrada em apenas um estudo $28 \%,{ }^{15}$ sendo que $50 \%$ foram por fratura de fêmur causando uma embolia e $50 \%$ por lesão neurológica causando um trauma intenso.

As características dos artigos selecionados estão descritas no quadro 1. 
Quadro1 - Consequências das quedas - artigos selecionados.

\begin{tabular}{|c|c|c|c|}
\hline Autor-Ano & Participantes & $\begin{array}{l}\text { Instrumentos de } \\
\text { Avaliação }\end{array}$ & Resultados - Consequências \\
\hline Ribeiro, AP, et al, 2008. & $\begin{array}{l}72 \text { idosos com idade } \\
\text { de } 60 \text { anos ou mais } \\
\text { em uma amostra de } \\
\text { conveniência, } \\
\text { moradores de uma } \\
\text { comunidade de baixa } \\
\text { renda do município } \\
\text { de Rio de Janeiro. }\end{array}$ & $\begin{array}{l}\text {-Questionário sobre a } \\
\text { situação } \\
\text { sociodemográfica, } \\
\text { quedas e qualidade de } \\
\text { vida. } \\
\text {-Escala padronizada de } \\
\text { avaliação da qualidade } \\
\text { de vida da OMS* } \\
\text { (WHOQOL) em sua } \\
\text { versão abreviada. }\end{array}$ & $\begin{array}{l}-24,3 \% \text { fraturas, sendo que } 33,3 \% \\
\text { de fêmur; } \\
-48,6 \% \text { atendimento médico; } \\
-88,5 \% \text { medo de cair; } \\
-25,9 \% \text { abandono de atividade; } \\
-23,1 \% \text { modificaram hábitos; } \\
\text { - } 19,2 \% \text { imobilização; } \\
-11,5 \% \text { lesão neurológica e } \\
\text { rearranjo familiar; } \\
-3,8 \% \text { mudaram de domicílio. }\end{array}$ \\
\hline Fabrício, SCC, et al, 2004. & $\begin{array}{l}50 \text { idosos de ambos } \\
\text { os sexos, de } 60 \text { anos } \\
\text { ou mais, com } \\
\text { residência fixa em } \\
\text { Ribeirão Preto (SP) } \\
\text { atendidos em duas } \\
\text { unidades de } \\
\text { internação de um } \\
\text { hospital } \\
\text { universitário. }\end{array}$ & $\begin{array}{l}\text {-Consulta ao } \\
\text { prontuário (nome, } \\
\text { idade, história de } \\
\text { queda, ocorrência de } \\
\text { internação). } \\
\text {-Entrevista estruturada } \\
\text { (identificação dos } \\
\text { sujeitos, história de } \\
\text { queda, mudanças } \\
\text { referidas pós } \\
\text { queda,restrições nas } \\
\text { ABVD* e a AIVD*). } \\
\text {-Avaliação de qualidade } \\
\text { de vida (Protocolo de } \\
\text { Yuaso \& Sguizzatto } \\
\text { elaborado á partir de } \\
\text { uma variação das } \\
\text { escalas de Katz e } \\
\text { Lawton. }\end{array}$ & 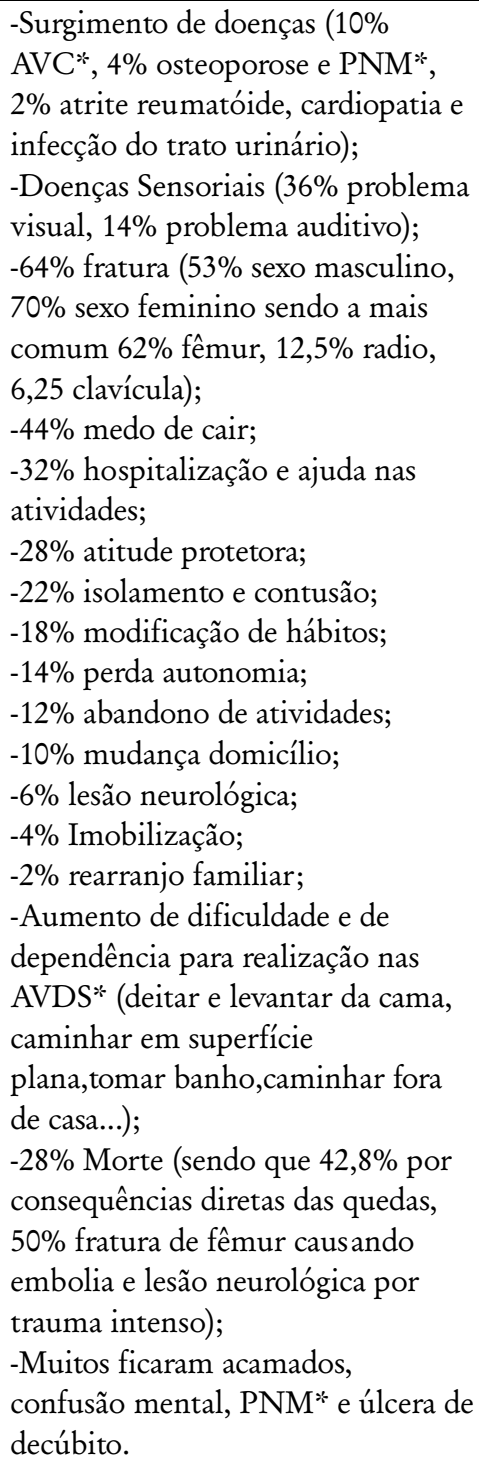 \\
\hline
\end{tabular}




\section{Quadro1 - Consequências das quedas - artigos selecionados. (Continuação)}

\begin{tabular}{|c|c|c|c|}
\hline Autor-Ano & Participantes & $\begin{array}{l}\text { Instrumentos de } \\
\text { Avaliação }\end{array}$ & Resultados - Consequências \\
\hline Gonzalez, CG, et al, 2001. & $\begin{array}{l}\text { Pessoas com } 60 \text { anos } \\
\text { ou mais que } \\
\text { espontaneamente } \\
\text { procuraram } \\
\text { atendimento médico } \\
\text { no programa para } \\
\text { Idoso (PUC-FOSIS- } \\
\text { Lo Espejo), durante } \\
\text { os meses de Janeiro a } \\
\text { maio de 1999, que } \\
\text { sofreram quedas nos } \\
6 \text { meses anteriores a } \\
\text { consulta. }\end{array}$ & $\begin{array}{l}\text {-Avaliação Funcional } \\
\text { (Escala Funcional da } \\
\text { Cruz Vermelha } \\
\text { Espanhola -EFCR). } \\
\text {-Presença de eventuais } \\
\text { perturbações de marcha } \\
\text { e equilíbrio (POMA*). } \\
\text {-Avaliação do Estado } \\
\text { de depressão (Teste de } \\
\text { Yesavage -versão } \\
\text { abreviada e validada da } \\
\text { escala de depressão } \\
\text { geriátrica- EDG). }\end{array}$ & $\begin{array}{l}-2,56 \% \text { fraturas; } \\
-Z \text { Zona de impacto: } 60 \% \text { perna e pé; } \\
-49 \% \text { braço e mão; } \\
-17 \% \text { cabeça; } \\
-14,7 \% \text { quadril; } \\
-8,3 \% \text { tronco; } \\
-2,6 \% \text { quadril; } \\
-10,2 \% \text { feridas; } \\
-1,3 \% \text { entorses; } \\
-21,2 \% \text { síndrome pós-queda; } \\
-12,2 \% \text { consulta médica. }\end{array}$ \\
\hline $\begin{array}{l}\text { Nachreiner ,NM, } \\
\text { et al, } 2007 .\end{array}$ & $\begin{array}{l}263 \text { mulheres que } \\
\text { viviam } \\
\text { independentes na } \\
\text { comunidade, Idade } \\
\text { entre } 70 \text { a } 99 \text { anos. } \\
\text { Ter uma queda no } \\
\text { mínimo como fator } \\
\text { de risco;escore } \\
\text { superior a } 23 \text { no } \\
\text { Mini-Mental; não ser } \\
\text { portadora de prótese } \\
\text { de MMII*; não } \\
\text { praticar exercícios } \\
\text { físicos regulamente; } \\
\text { ser capaz de ler e } \\
\text { entender inglês por } \\
\text { telefone e } \\
\text { disponíveis para } \\
\text { acompanhamento. }\end{array}$ & $\begin{array}{l}\text {-Calendário de quedas } \\
\text { (número e horário de } \\
\text { quedas). } \\
\text { - Enfermeira coletou } \\
\text { informações sobre as } \\
\text { quedas por telefone. }\end{array}$ & $\begin{array}{l}-30 \% \text { lesões de tecidos moles; } \\
-9 \% \text { abrasões; } \\
-5 \% \text { fraturas e lacerações sem } \\
\text { suturas respectivamente, fraturas } \\
\text { mais frequentes foram de quadril } \\
(\mathrm{N}=5) \text { e MMSS* }(\mathrm{N}=5) ; \\
-7 \% \text { procuraram um serviço de } \\
\text { emergência; } \\
-10 \% \text { foram capazes de voltar à } \\
\text { independência não imediatamente; } \\
-20 \% \text { necessitaram de algum tipo de } \\
\text { ajuda; } \\
-5 \% \text { assistência nas AVDS*. }\end{array}$ \\
\hline Berg, WR,et al 1997. & $\begin{array}{l}96 \text { voluntários ( } 38 \\
\text { homens, } 58 \\
\text { mulheres). } \\
\text { Entre } 60 \text { a } 88 \text { anos, } \\
\text { que vivem } \\
\text { independente na } \\
\text { comunidade e serem } \\
\text { capazes de andar sem } \\
\text { ajuda. }\end{array}$ & $\begin{array}{l}\text {-Testes e questionários } \\
\text { abordando o estado de } \\
\text { saúde, visão, atividade } \\
\text { física e o desempenho e } \\
\text { história de quedas. } \\
\text {-Cartão - relatório } \\
\text { (calendário de quedas } \\
\text { relatando data e } \\
\text { horário). } \\
\text { - Questionário } \\
\text { adaptado a partir de } \\
\text { uma pesquisa } \\
\text { desenvolvida no Center } \\
\text { for Health Research, } \\
\text { que descreve a queda } \\
\text { propriamente dita, } \\
\text { quando e onde ocorreu } \\
\text { a queda, as atividades } \\
\text { que precederam a } \\
\text { queda, sensações físicas } \\
\text { imediatamente } \\
\text { anteriores à } \\
\text { queda e prejuízos } \\
\text { resultantes. }\end{array}$ & $\begin{array}{l}-5 \% \text { fraturas ( } 2 \text { fraturas em quadril, } \\
1 \text { no cotovelo, } 1 \text { nos pés e } 1 \text { nas } \\
\text { costelas, todas no sexo feminino); } \\
-9 \% \text { resultaram em lesões dos } \\
\text { tecidos moles (cortes e contusões); } \\
-31 \% \text { resultaram em dor (duração } \\
\text { de } 2 \text { dias ou mais); } \\
-21 \% \text { resultaram em dor (duração } \\
\text { de } 5 \text { dias ou mais); } \\
-64 \% \text { mudança de comportamento } \\
\text { e ambiente. }\end{array}$ \\
\hline
\end{tabular}


Quadro1 - Consequências das quedas - artigos selecionados. (Continuação)

\begin{tabular}{|c|c|c|c|}
\hline Autor-Ano & Participantes & $\begin{array}{l}\text { Instrumentos de } \\
\text { Avaliação }\end{array}$ & Resultados - Consequências \\
\hline Johansson B, 1998. & $\begin{array}{l}\mathrm{N}=1639 . \text { As } \\
\text { mulheres } \\
\text { representavam } 70 \% \\
\text { da amostra. } \\
\text { Idosas acima de } 65 \\
\text { anos que viviam em } \\
\text { suas próprias casas. } \\
\text { Foram excluídas } \\
\text { pessoas que viviam } \\
\text { em serviço de } \\
\text { habitação da } \\
\text { comunidade } \\
\text { (Institucionalizados). }\end{array}$ & $\begin{array}{l}\text {-Ficha de inscrição } \\
\text { (idade, sexo, outros } \\
\text { dados da identidade, } \\
\text { data e hora da lesão e } \\
\text { da consulta com o } \\
\text { médico, o local e área } \\
\text { onde ocorreu o } \\
\text { acidente e a causa da } \\
\text { lesão. Atividade da } \\
\text { vítima e estrutura do } \\
\text { movimento em relação } \\
\text { com o acidente } \\
\text { Influência alcoólica; } \\
\text { Tipo de lesão e medidas } \\
\text { tomadas). }\end{array}$ & $\begin{array}{l}-11,6 \% \text { fratura de quadril; } \\
-9,9 \% \text { fraturas de antebraço; } \\
-9,7 \% \text { contusões com pele intacta; } \\
-7,4 \% \text { feridas na cabeça; } \\
-7 \% \text { fratura de braço (as fraturas } \\
\text { representaram } 46,4 \% \text { das lesões); } \\
\text { Mais frequente entre mulheres } \\
(51,3 \%) \text { do que em homens }(34,1 \%) \text {. }\end{array}$ \\
\hline Vianda, SS, et al, 2004. & $\begin{array}{l}\text { Uma amostra } \\
\text { aleatória de estudo } \\
\text { longitudinal Aging } \\
\text { Amsterdam (LASA), } \\
204 \text { participantes } \\
\text { (112 mulheres e } 92 \\
\text { homens), com } 65 \\
\text { anos ou mais que } \\
\text { tenham caído no ano } \\
\text { anterior à entrevista. }\end{array}$ & $\begin{array}{l}\text {-Declínio de Status } \\
\text { funcional (questionário } \\
\text { sobre o grau de } \\
\text { dificuldade com } \\
\text { atividades funcionais). } \\
\text {-Depressão (Centro de } \\
\text { estudos } \\
\text { epidemiológicos- escala } \\
\text { de depressão -CES-D) } \\
\text {-Prejuízo cognitivo } \\
\text { (Mini-Mental). } \\
\text {-Questionário LAPAQ } \\
\text { (LASA Physical } \\
\text { Activity } \\
\text { Questionnaire), com } \\
\text { testes de desempenho. }\end{array}$ & 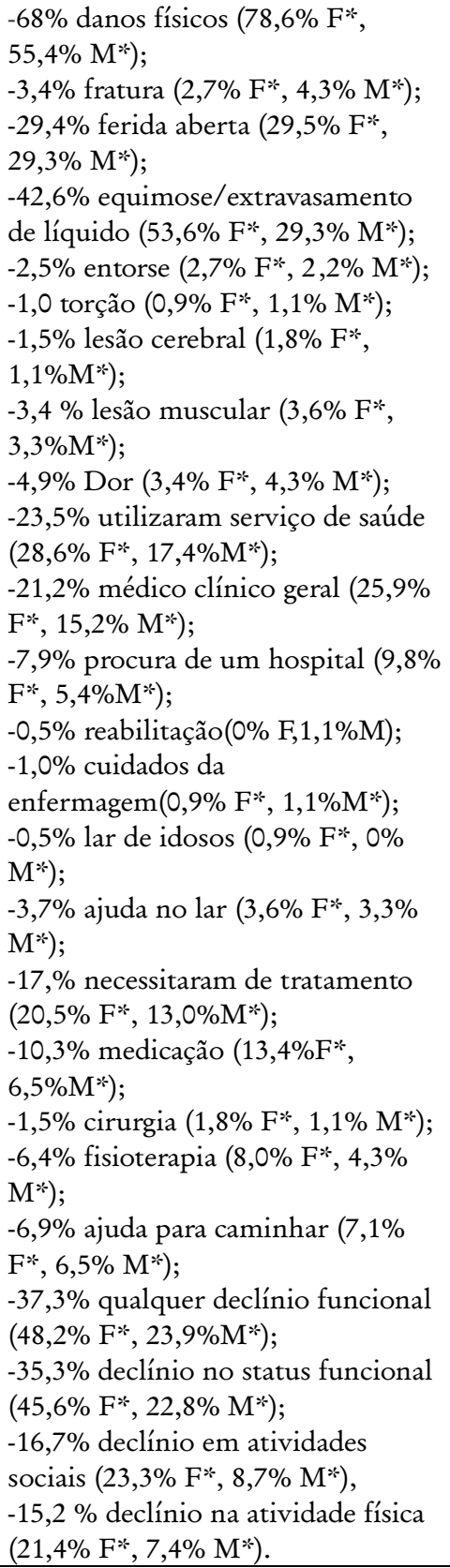 \\
\hline
\end{tabular}




\section{Quadro1 - Consequências das quedas - artigos selecionados. (Continuação)}

\begin{tabular}{|c|c|c|c|}
\hline Autor-Ano & Participantes & $\begin{array}{l}\text { Instrumentos de } \\
\text { Avaliação }\end{array}$ & Resultados - Consequências \\
\hline Varas, FF, et al, 2006. & $\begin{array}{l}362 \text { pessoas com } \\
\text { mais de } 70 \text { anos } \\
\text { residentes na } \\
\text { comunidade (mais de } \\
6 \text { meses), em três } \\
\text { áreas básicas de saúde } \\
\text {-ZBS da cidade de } \\
\text { Córdoba. } \\
\text { Foram excluídos do } \\
\text { estudo: doentes } \\
\text { terminais com } \\
\text { esperança de vida } \\
\text { menor que } 6 \\
\text { meses,portadores de } \\
\text { algum distúrbio } \\
\text { psiquiátrico e } \\
\text { pacientes } \\
\text { institucionalizados. }\end{array}$ & $\begin{array}{l}\text {-Questionário da } \\
\text { OMS* (Quedas em } \\
\text { idosos). } \\
\text {-Instrumento genérico } \\
\text { de avaliação de } \\
\text { qualidade de vida } \\
\text { (EUROQOL-5D), que } \\
\text { avalia } \\
\text { mobilidade,cuidados } \\
\text { pessoais, AVD's*, dor, } \\
\text { ansiedade, depressão e } \\
\text { desconforto. } \\
\text {-Caderno de coleta de } \\
\text { dados (idade, questões } \\
\text { sócio-demográficas, } \\
\text { estado civil, } \\
\text { escolaridade, profissão, } \\
\text { avaliação clínica e } \\
\text { funcional, AVDS*, } \\
\text { doenças crônicas, } \\
\text { características das } \\
\text { quedas, consequências, } \\
\text { tipo de contato com o } \\
\text { sistema de saúde). }\end{array}$ & $\begin{array}{l}-56 \% \text { feridas superficiais; } \\
-7,8 \% \text { fraturas; } \\
-25 \% \text { mudança na vida; } \\
-44,7 \% \text { medo de voltar a cair; } \\
-22 \% \text { limitação no grau de } \\
\text { mobilidade; } \\
-40,9 \% \text { dificuldade de andar; } \\
-51 \% \text { dificuldade para utilizar os } \\
\text { MMSS*; } \\
-40,2 \% \text { dificuldade de levantar da } \\
\text { cadeira; } \\
-34,5 \% \text { dificuldade de realizar } \\
\text { exercícios; } \\
-39,4 \% \text { problema de memória; } \\
-51,2 \% \text { problema para se orientar } \\
\text { no tempo; } \\
-58,8 \% \text { problema para se orientar } \\
\text { no espaço; } \\
-44,7 \% \text { problema de compreensão; } \\
-45,7 \% \text { enfermidade neurológica; } \\
-42,5 \% \text { enfermidade nos pés; } \\
-51,7 \% \text { problemas para realizar } \\
\text { cuidados pessoais; } \\
-46,5 \% \text { problema para realizar } \\
\text { atividade cotidiana. }\end{array}$ \\
\hline Wilkins K, 1999. & $\begin{array}{l}2081 \text { pessoas com } \\
\text { mais de } 65 \text { anos em } \\
1994 \text { / } 95 . \text { Para os } \\
\text { quais os dados } \\
\text { estavam disponíveis } \\
\text { e que ainda estavam } \\
\text { vivos em } 1996 \text { / } 97 . \\
\text { Corte transversal de } \\
\mathrm{N}=11.282 \text { nesta } \\
\text { faixa etária em } 1996 \\
\text { / } 97 \text { resultando em } \\
\text { um total de } \mathrm{N}= \\
13.363 . \\
\text { Idosos do Canadá e } \\
\text { províncias } \\
\mathrm{N}=822 \text { Homens e } \\
\mathrm{N}=1259 \text { Mulheres. }\end{array}$ & $\begin{array}{l}\text { Este artigo é baseado na } \\
\text { Statistics Canada's } \\
\text { National Population } \\
\text { Health Survey ( } \\
\text { NPHS), que possue } \\
\text { componentes } \\
\text { tranversais e } \\
\text { longitudinais } \\
\text { conduzido por } \\
\text { estatísticas Canadenses. }\end{array}$ & $\begin{array}{l}-49 \% \text { fraturas; } \\
-30 \% \text { pernas e pés; } \\
-12 \% \text { quadril; } \\
-25 \% \text { braços e mãos; } \\
-11 \% \text { foram hospitalizados. }\end{array}$ \\
\hline Kong, SK, et al, 2002. & $\begin{array}{l}20 \text { chineses ( } 5 \\
\text { homens e } 15 \\
\text { mulheres), com } 65 \\
\text { anos ou mais (média } \\
\text { de idade de } 82 \text { anos), } \\
\text { em um hospital de } \\
\text { Hong Kong que } \\
\text { sofreram quedas } 48 \\
\text { horas antes. }\end{array}$ & $\begin{array}{l}\text {-Entrevista guia que } \\
\text { abrange três tópicos } \\
\text { (sobre os sentimentos } \\
\text { experiência de queda, } \\
\text { preocupações com } \\
\text { queda, e } \\
\text { comportamento após a } \\
\text { queda). }\end{array}$ & $\begin{array}{l}\text {-18 a } 20 \text { idosos tiveram sentimento } \\
\text { de impotência; } \\
\text {-10 idosos tiveram medo de cair; } \\
\text {-Perda da liberdade pessoal e } \\
\text { independência; } \\
\text {-Imobilização; } \\
\text {-Procura de atendimento; } \\
\text {-Tristeza. }\end{array}$ \\
\hline \multicolumn{4}{|c|}{$\begin{array}{l}\text { Abreviações: } \\
\text { "AVC-Acidente Vascular Cerebral } \\
\text { "PNM-Pneumonia } \\
\text { "ABVD- Atividades Básicas da Vida Diária } \\
\text { "AIVD- Atividades Instrumentais da Vida Diária } \\
\text { "MMSS-Membros Superiores } \\
\text { "MMII-Membro Inferiores }\end{array}$} \\
\hline
\end{tabular}




\section{DISCUSSÃO}

De acordo com os artigos incluídos nesta revisão, as fraturas (quadril, fêmur, braço, antebraço, perna e pé) estão entre as consequências mais citadas. Elas foram mencionadas em todos os estudos, com exceção de um artigo (Kong et al. ${ }^{32}$ ), que avaliou apenas desfechos psicossociais. Dentre as fraturas, a mais citada foi a de quadril ou fêmur.

A ocorrência das fraturas variou muito entre os estudos. No estudo de Berg et al., ${ }^{28}$ das 91 quedas que ocorreram, apenas cinco resultaram em fratura. No entanto, o estudo de Johansson et al. ${ }^{29}$ mostra que as fraturas foram responsáveis por cerca da metade dos registros $(\mathrm{N}=1.639)$ e o estudo de Fabrício et al. ${ }^{15}$ cita uma frequência de $64 \%$ de fraturas após as quedas.

Essa variação tão grande entre os estudos em relação à ocorrência das fraturas pode ter ocorrido devido aos diferentes métodos de investigação das fraturas e às diferentes populações estudas. A amostra do estudo de Fabrício et al. ${ }^{15}$ era composta por idosos que haviam sido atendidos em uma unidade de internação na cidade de Ribeirão Preto, por causas externas de traumatismo acidental. De forma similar, no estudo de Johansson et al..$^{29}$ foram utilizados apenas os registros de casos de acidentes em idosos que necessitaram de atenção médica.

Assim, observa-se que nesses estudos os idosos que caíram, mas não necessitaram de atendimento médico, não fizeram parte do estudo. Só foram incluídos os idosos que procuraram algum serviço de saúde após a queda, o que pode justificar a alta prevalência de fraturas nesses trabalhos. Por outro lado, Berg et al. ${ }^{28}$ investigaram um grupo de idosos prospectivamente por um ano e registraram todas as ocorrências, independentemente de ter necessitado de atendimento médico.

Os estudos avaliados demonstraram que a ocorrência de fraturas parece ser maior nas mulheres do que nos idosos do sexo masculino. ${ }^{15,26-29}$ Esta maior propensão das idosas às fraturas ocorre provavelmente devido à existência de osteoporose. Este achado traz importantes implicações para as políticas de saúde, uma vez que esta doença pode ser diagnosticada, tratada $\mathrm{e}$ prevenida, sendo que na maioria dos casos a prevenção não é dispendiosa. Assim, considerando os prejuízos trazidos pelas fraturas para a saúde dos idosos, é importante que sejam implementados programas de prevenção e tratamento dessa doença. ${ }^{33}$

Além de estarem mais propensas a apresentar fraturas, alguns estudos confirmam que a frequência de quedas é maior também em mulheres idosas do que em homens e que a maioria das quedas ocorrem dentro do próprio domicilio ou ao redor da casa. ${ }^{27,28}$ Segundo Berg et al., ${ }^{28}$ essa diferença pode estar ligada a vários fatores, como: idade mais avançada, frequência diminuída de atividades externas, utilização de grande quantidade de drogas, uso de psicotrópicos e diminuição de força de preensão.

Além das fraturas, outra consequência bastante relacionada com as quedas foram as lesões de tecidos moles. De acordo com Berg et al., ${ }^{28} 8 \%$ das quedas resultaram em lesões de tecidos moles. Nachreiner et al. ${ }^{27}$ demonstraram uma frequência ainda maior, sendo que $30 \%$ das quedas geraram algum tipo de dano tecidual no idoso e $9 \%$ foram seguidas de abrasões. A alta frequência de lesões pode ser explicada pelo fato de o idoso ser mais vulnerável fisiologicamente, mas isso não significa que a lesão seja inevitável.

Em relação às hospitalizações, em 2005 ocorreram 61.368 hospitalizações por queda de pessoas com 60 anos ou mais, de ambos os sexos, representando $2,8 \%$ de todas as internações de idosos no país e $54,4 \%$ das internações por lesões e envenenamentos neste grupo etário. ${ }^{25}$ Dos dez artigos utilizados para esta revisão sete ${ }^{15,25-27,30-32}$ constataram que a queda levou os idosos a procurarem algum tipo de atendimento médico ou hospitalização. Nachereiner et al. ${ }^{27}$ relataram que $7 \%$ das quedas resultaram em visitas em serviços de urgência e $9 \%$ das quedas resultaram 
em atendimento ambulatorial. Já no estudo de Vianda et al., ${ }^{30} 23,5 \%$ dos idosos que sofreram quedas recorreram ao serviço de saúde. Este achado está semelhante ao de Johansson et al., ${ }^{29}$ no qual $20 \%$ dos casos de quedas necessitaram de visitas adicionais ao médico e $24,5 \%$ necessitaram de internação.

Esses dados demonstram o impacto que as quedas têm para os sistemas público e privado de saúde. Além disso, eles estão de acordo com os dados que demonstram os altos custos que as quedas representam para os governos, ${ }^{22}$ apontando a necessidade de políticas e programas de prevenção desta grande síndrome geriátrica.

Como esperado, as quedas não geraram apenas repercussões físicas nos idosos. O medo de cair foi uma consequência destacada por vários autores ${ }^{15,20,25,26,32}$ e está relacionado com qualidade de vida do idoso. O medo após a queda pode trazer consigo não somente o medo de novas quedas, mas também de machucar-se, ser hospitalizado, sofrer imobilizações, ter declínio de saúde e tornar-se dependente de outras pessoas para o autocuidado ou para realizar atividades $\mathrm{da}$ vida diária. ${ }^{32}$ Por outro lado, o medo pode atuar também como um fator protetor na medida em que o idoso, em função dele, adota comportamentos preventivos. ${ }^{15}$ Segundo Berg et al., ${ }^{28}$ o medo de cair foi mais prevalente nas mulheres e tem sido associado à redução de atividades diárias.

A restrição de atividades é uma das consequências mais prejudiciais que o medo de cair pode levar, uma vez que pode contribuir para o declínio funcional e aumentar o risco de uma nova queda. Ribeiro et al. ${ }^{25}$ relataram que $25,9 \%$ dos idosos que caíram restringiram suas atividades diárias após a queda. A restrição de atividades pode ocorrer tanto por medo de expor-se ao risco de queda, como por atitudes protetoras da sociedade, familiares e cuidadores. As pessoas podem passar a ver o idoso que cai como frágil, e muitas vezes afastá-lo de tais atividades. ${ }^{15}$ Assim, a partir dos danos físicos, começa uma reação em cadeia que leva a danos psicológicos, sociais e econômicos.
Outra importante consequência citada foi o declínio funcional. Varas et al..$^{20}$ encontraram que $22 \%$ dos idosos que caíram tiveram alguma limitação no grau de mobilidade. De acordo com Fabrício et al., ${ }^{15}$ levantar-se da cama, tomar banho e subir escadas foram as principais atividades que sofreram impacto após a queda. Considerando-se a importância da capacidade funcional para independência e qualidade de vida dos idosos e seus familiares, ${ }^{34}$ a alta frequência do declínio funcional após as quedas se torna preocupante e um indicador da necessidade de programas que busquem a prevenção de tal declínio.

Como mencionado anteriormente, as consequências das quedas não abrangem somente os idosos que caem, mas também sua família. $\mathrm{O}$ rearranjo familiar estava presente em um dos artigos encontrados. ${ }^{15} \mathrm{O}$ aumento da dependência do idoso após a queda pode ser uma explicação para este achado. Entende-se que essa mudança também ocorre devido aos altos custos e mudança na rotina de toda família, pois a família deverá realizar modificações na estrutura física do domićlio, para que o idoso possa ter melhor qualidade de vida, diminuindo assim os riscos de novas quedas, além de arcar com tratamentos complementares.

A morte foi citada em apenas um dos dez artigos selecionados, ${ }^{15}$ tendo como um total de $28 \%$ dos idosos, sendo que $42,8 \%$ ocorreram em menos de um ano por consequência direta da queda. Dentre as causas de óbito, destaca-se a fratura de fêmur causando embolia e lesão neurológica por trauma intenso. $\mathrm{O}$ número elevado de mortes devido à queda neste estudo pode ser explicado pela forma como os idosos foram recrutados para o estudo. Só participaram idosos que buscaram atendimento médico devido a traumas. Assim, a amostra do estudo foi composta pelos casos mais graves de quedas.

Os resultados deste estudo demonstraram que as consequências das quedas são variadas e vão além das tão citadas fraturas e do medo de cair. Considerando a gravidade de várias destas consequências, é necessário que sejam elaborados e implementados programas eficazes de prevenção 
das quedas. Foi demonstrado, ainda, que algumas consequências das quedas podem levar a outras repercussões que podem aumentar ainda mais o risco do idoso cair, como é o caso do medo de quedas levando à restrição de atividade. Assim, fica evidente que, além dos programas de prevenção às quedas, é importante também a realização de programas de reabilitação após as quedas, com o objetivo de impedir ou minimizar a ocorrência de tais repercussões.

\section{CONCLUSÃO}

A partir deste estudo, pode-se concluir que a ocorrência de quedas em idosos pode resultar em um relevante problema de saúde. Com base na revisão literária realizada, constatou-se que uma

\section{REFERÊNCIAS}

1. Guimarães LHCT, et al. Comparação da propensão de quedas entre idosos que praticam atividade física e os idosos sedentários. Rev Neuroc 2004; 12 (2):3 [acesso em 2008 mar 20]. Disponível em: http://www.unifesp.br/dneuro/ neurociencias/vol12_2/ quedas.htm.

2. Kauffman, TL. O Indivíduo como um todo: manual de reabilitação geriátrica. Rio de Janeiro: Ganabara Koogan; 2001. 416 p.

3. Duarte YAO. O processo de envelhecimento e a assistência ao idoso. In: Manual de enfermagem: programa saúde da família. São Paulo : Ministério da Saúde ; 2001.p.185 - 196.

4. Costa EFA, Porto CC, Almeida JC, Cipullo JP, Martin JFV. Semiologia do Idoso. In: Porto CC. Semiologia Médica. 4. ed. Rio de Janeiro: Guanabara-Koogan ; 2001.1316 p.

5. Siqueira RL, Botelho MIV, Coelho FMG. A velhice: algumas considerações teóricas e conceituais. Ciênc Saúd Coletiva 2002; 7(4):899906.

6. Siqueira FV, Facchini LA, Piccini RX, Silveira DS, Vieira V, Halla PC, et al. Prevalência de quedas em idosos e fatores associados. Rev Saúde Pública 2007;41(5):749-56.

7. Instituto Brasileiro de Geografia e Estatística (IBGE). Pesquisa nacional por amostra de domicílio : censo demográfico 1991 e 2000 e contagem populacional. [Acesso 22 out 2006]. grande variedade de consequências pode ocorrer após um episódio de queda. Estas podem envolver danos físicos, como lesões teciduais, ferimentos e fraturas, declínio funcional e aumento da dependência e questões psicossociais, como medo de cair, isolamento e perda da autonomia.

Considerando a gravidade de várias destas consequências, há necessidade de programas eficazes de prevenção das quedas. Além destes, também é importante a implantação de programas de reabilitação após as quedas, com o objetivo de impedir ou minimizar a ocorrência de tais repercussões.

Ficou demonstrado também que as quedas podem trazer importantes implicações para a família desse idoso e para a sociedade.

Disponível em: URL: http:// tabnet.datasus.gov.br/cgi/idb2004/a1 5uf.htm.

8. Walker JE, Howland J. Falls and fear of falling among elderly persons living in the community: the occupational therapy interventions. Am J Occupational Therapy 1991; 45(2): 119-22.

9. Murphy SL, Williams CS, Gill TM. Characteristics associated with fear of falling and activity restriction in community-living older persons. J am can geriatr society 2002; 50(3): 516-20.

10. Tinetti ME. Prevention of falls and fall injuries in elderly persons: a research agenda. Prev Med 1994; 23(5): 756-62.

11. Moura RN,Santos FC, Driemeier M, Santos LM, Ramos LR. Quedas em idosos: fatores de risco associados. Gerontologia 1999 ; 7(2):15-21.

12. Carvalhaes N, et al. Quedas. In: 1 Congresso paulista de Geriatria e Gerontologia ;1998 jun 24- 27 de junho de 1998; São Paulo, Brasil. Consensos de gerontologia, São Paulo: Sociedade Brasielira de Geriatria Gerontologia 1998. p. 5-18

13. Tinetti ME. Preventing falls in elderly persons. N Engl J Méd 2003; 348(1): 42-9.

14. Liu-Ambrose $\mathrm{T}$, et al. Resistance and agility training reduce fall risk in women aged 75 to 85 with low bone mass: a 6-month randomized, controlled trial. J Am Geriatr Soc 2004; 52:1-9. 
15. Fabrício SCC, Rodrigues RAP, Junior MLC. Causas e conseqüências de quedas em idosos atendidos em hospital público. Rev Saúde Públ 2004; 38(1): 93-9.

16. Rubenstein LZ, Powers C, Maclean CH. Quality Indicators for the management and prevention of fall and mobility problems in vulnerable elders. Ann Intern Méd 2001; 135(8): 686-93.

17. Cumming RG. Intervention strategies and riskfactor modification for falls prevention: a review of recent interventions studies. Clinics Geriatric Mede 2002; 18(2) : 175-89

18. Hogan DB, Hogan DB, MacDonald FA, Betts JBS, Ebly EM, Delarue B,, et al. A ramdomized controlled trial of a community-based consultation service to prevent falls. Can med Ass Journal 2001 ; 165 (5): 537-543.

19. Tinetti ME, Baker DI, Mcavay G, et al. A multifactorial intervention to reduce the risk of falling among elderly people living in the community. N Engl Journal Méd 1994; 331: 821-7.

20. Varas-Fabra F, Castro ME, Torres LAP, Fernández MJF, Moral RR, Berge IE. Caídas en ancianos de la comunidad: prevalencia, consecuencias y factores asociados. Aten Primaria 2006 nov; 38 (8): 450-5.

21. Perracini MR, Ramos LR. Fatores associados a quedas em uma coorte de idosos residentes na comunidade. Rev Saúde Pública 2002; 36(6): 709-16

22. Mello-Jorge MHPD, Koizumi MS. Gastos governamentais do SUS com internações hospitalares por causas externas: análise no Estado de São Paulo 2000. Rev Bras Epidemiol 2004 jun; 7(2): 228-238.

23. Meio Norte.com.Cresce gastos do SUS com queda de idosos.[Acesso em 18 abril 2008]. Disponível em: URL: http:// www.meionorte.com/noticias, Cresce-gastos-doSUS-com-queda-de-idosos, $45309 . \mathrm{html}$.
24. Carvalho AM, Coutinho ESF. Demência como fator de risco para fraturas graves em idosos. Rev Saúd Pública 2002; 36 (4): 448-54.

25. Ribeiro AP, Souza ER, Atie S, Souza AC, Schilithz AO.A influência das quedas na qualidade de vida de idosos. Ciênc saúd coletiva 2008 ago; 13(4): 1265-1273.

26. Gonzalez C, Marin LG, Cardoso PP, Pereira GZ. Características de las caídas en el adulto mayor que vive en la comunidad. Rev méd Chile 2001 set ; 129(9):1021-1030.

27. Nachreiner Nm, Findorff Mj, Wyman Jf, Mccarthy Tc. Circumstances and consequences of falls in community-dwelling older women. J. Womes Health 2007 dec ; 16 (10):1437-46.

28. Berg Wp, Alessio Hm, Mills Em, Tong C. Circunstances and consequences of falls in independent community-dwelling older adults. Age Agein 1997 jul; 26(4):261-8.

29. Johansson B. Fall injuries among elderly persons living at home.Scand J Caring Sci 1998; 12 (2):67-72.

30. Vianda S, Stel,JH, Smit SMF, Pluijm PL. Consequences of falling in older men and women and risk factors for health service use and functional decline. Age Agein 2004 jan; 33(1):58-65.

31. Wilkins Kathryn. Health care consequences of falls for seniors. Health Rep 1999; 10(4): 47-55.

32. Kong Ks, Lee Fk, Mackenzie Ae, Lee Dt. Psychosocial consequences of falling: the perspective of older Hong Kong chinese who had experienced recent falls. J Adv Nurs 2002 feb; 37(3):234-42.

33. Gawryszewski VP, Koizumi MS; Mello-Jorge MHP. Mortes e internações por causas externas entre os idosos no Brasil : o desafio de integrar a saúde coletiva e atenção Individual. Rev Assoc Med Brás 2004; 50(1): 97-103.

34. Gama ZAS, Conesa AG, Ferreira MS. Epidemiología de caídas de ancianos en España: una revisión sistemática. Rev Esp Salud Pública 2008; 82(1): 43-56. 